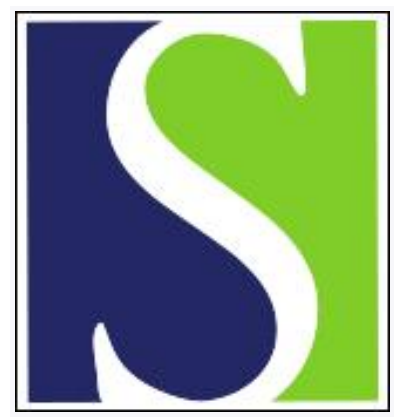

Scand J Work Environ Health 2016;42(6):481-489

https://doi.org/10.5271/sjweh.3600

Published online: 13 Oct 2016, Issue date: 01 Jun 2016

Sickness absence and permanent work disability in relation to upper- and lower-body pain and occupational mechanical and psychosocial exposures

by Sommer TD, Svendsen SW, Frost P

Musculoskeletal pain in multiple sites has been associated with sickness absence and permanent work disability. We found that high occupational mechanical exposures were associated with sickness absence, particularly in case of combined pain in the upper and lower body. We also found that combined pain and low social support at work were associated with permanent work disability.

Affiliation: Danish Ramazzini Centre, Department of Occupational Medicine - University Research Clinic, Regional Hospital West Jutland, Gl. Landevej 61, 7400 Herning, Denmark. Tine.g.sommer@gmail.com

Refers to the following texts of the Journal: $2014 ; 40(1): 82-88$ 2013;39(6):568-577 2012;38(6):582-589 2010;36(1):34-41

The following articles refer to this text: 2017;43(5):393-395;

2017;43(5):415-425; 2020;46(3):268-277; 2022;48(2):137-147

Key terms: cohort study; control; demand; force; job exposure matrix; lifting; lower-body pain; occupational exposure; occupational mechanical exposure; pain; permanent work disability; posture; psychosocial exposure; repetition; sickness absence; sickness absence; social support; upper-body pain; work disability

This article in PubMed: www.ncbi.nlm.nih.gov/pubmed/27779638

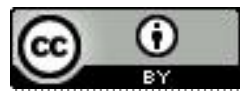




\title{
Sickness absence and permanent work disability in relation to upper- and lower-body pain and occupational mechanical and psychosocial exposures
}

\author{
by Tine Gjedde Sommer, MSc, ${ }^{1}$ Susanne Wulff Svendsen, PhD, ${ }^{1}$ Poul Frost, PhD ${ }^{2}$
}

\begin{abstract}
Sommer TG, Svendsen SW, Frost P. Sickness absence and permanent work disability in relation to upper- and lower-body pain and occupational mechanical and psychosocial exposures. Scand J Work Environ Health. 2016;42(6):481-489. doi:10.5271/sjweh.3600
\end{abstract}

\begin{abstract}
Objective The aim of this study was to examine if combined pain (pain in the upper and lower body), high occupational mechanical exposures, and job strain predict sickness absence and permanent work disability, particularly if co-occurring.

Methods This longitudinal study was based on the musculoskeletal research database at the Danish Ramazzini Centre. We linked baseline information from 2001-2004 on musculoskeletal pain, job titles, and covariates with register information on sickness absence and permanent work disability. Mechanical and psychosocial exposure estimates were obtained from job exposure matrices. We used multivariable Cox regression.

Results The study included 25292 persons. Compared to low occupational mechanical exposures and no pain, low mechanical exposures and combined pain showed an adjusted hazard ratio $\left(\mathrm{HR}_{\mathrm{adj}}\right)$ of $1.81[95 \%$ confidence interval (95\% CI) 1.58-2.08], while high mechanical exposures and combined pain showed $\mathrm{HR}_{\mathrm{adj}} 3.71(95 \% \mathrm{CI}$ 3.24-4.25) for sickness absence. The corresponding values for permanent work disability were 3.35 (95\% CI 1.74-6.45) and 4.59 (95\% CI 2.36-8.94). Job strain showed an association with sickness absence [HR adj $_{1.24}$ (95\% CI 1.10-1.39)], while low social support was associated with both sickness absence and permanent work disability [HR adj 1.30 (95\% CI 1.19-1.41) and 2.19 (95\% CI 1.41-3.41), respectively].

Conclusions Combined pain in the upper and lower body was associated with sickness absence and permanent work disability, and high occupational mechanical exposures further increased the risk. Job strain showed a modest association with sickness absence, while low social support at work was associated with both outcomes, especially permanent work disability.
\end{abstract}

Key terms cohort study; control; demand; force; job exposure matrix; lifting; occupational exposure; posture; repetition; social support.

Musculoskeletal pain in multiple sites is common in working populations $(1,2)$ and has been found to be a risk factor for sickness absence (3-9). In Denmark, approximately $25 \%$ of sickness absence periods $>8$ weeks and $20 \%$ of allocated disability pensions are related to musculoskeletal disorders (10).

In a working population, we recently found that combined pain (pain in the upper and lower body) was more prevalent than pain in either the upper or lower body alone (11). Our results also suggested that local effects of occupational mechanical exposures on both the upper and the lower body explained combined pain to some extent. Irrespective of musculoskeletal pain, the risk of sickness absence has been reported to increase with occupational mechanical exposures, such as work with arms elevated $(12,13)$, repetitive work movements (14), and physically hard work $(14,15)$. In a study of employees with neck-shoulder and/or low-back pain, physically hard work was also associated with sickness absence (16); the study did not examine if the influence of physically hard work differed between employees with pain in one or both regions. Furthermore, physically hard work has been associated with sickness absence due to musculoskeletal pain (17). Occupational mechanical exposures have been linked to both all-cause disability pension $(18,19)$ and disability pension due to musculoskeletal causes (19).

1 Danish Ramazzini Centre, Department of Occupational Medicine, Regional Hospital West Jutland - University Research Clinic, Herning, Denmark.

2 Danish Ramazzini Centre, Department of Occupational Medicine, Aarhus University Hospital, Aarhus, Denmark.

Correspondence to: Tine Gjedde Sommer, Danish Ramazzini Centre, Department of Occupational Medicine - University Research Clinic, Regional Hospital West Jutland, Gl. Landevej 61, 7400 Herning, Denmark. [E-mail: Tine.g.sommer@gmail.com]. 
Adverse psychosocial factors in terms of high job demands or low job control have been associated with sickness absence $(14,20)$, although inconsistently $(13$, 21). Among workers at an aluminum plant, both high job strain (a combination of high job demands and low job control) and pain in more than two sites were associated with sickness absence (20). Another study has found that high job strain - assessed using a job exposure matrix was significantly associated with disability pension due to musculoskeletal causes (22).

A recent study examined if co-occurrence of physical workload or adverse psychosocial factors (high job demands, low job control, or low social support) and multisite musculoskeletal pain predicted an excessive risk of sickness absence (23). Associations between multisite pain and sickness absence were found both for employees with high physical or psychosocial exposures and employees without, but the exposures did not seem to modify the associations. For disability pension, we have been unable to identify studies that have examined the combined influence of occupational mechanical or psychosocial exposures and musculoskeletal pain in more than one site.

The present study examined the hypothesis that combined pain is a predictor of sickness absence and permanent work disability when compared to no pain, with intermediate risk estimates related to pain limited to either the upper or the lower body, and that the risk estimates are further increased in case of high occupational mechanical exposures and job strain, respectively.

\section{Methods}

We conducted a longitudinal study using baseline data from the musculoskeletal research database (MRD) at the Danish Ramazzini Centre. The MRD contains questionnaire data from 39590 participants in one or more of nine previous epidemiological studies of musculoskeletal symptoms. The original studies have been summarized elsewhere (24). The Danish Data Protection Agency approved the establishment of the MRD and the present study (J.no. 2009-41-3431).

\section{Study cohort}

We excluded four of the nine studies in the MRD: one study did not have information on lower body pain and three studies were conducted before register information on sickness absence and permanent work disability was available. Thus, the present study included data from the (i) neck and upper-extremity disorders among technical assistants (NUDATA) study, (ii) project on work at institutions for adults with disabilities, (iii) project on clean- ers and shop assistants, (iv) FAUST study of employees at 39 work sites, and (v) ASUSI study of a working population sample (24). These studies were conducted from 2001 to 2004. Participants were included if they were $18-<65$ years old at baseline and had completed the questions on musculoskeletal pain. We excluded participants without information on occupational title and participants who received transfer payments due to sickness absence or permanent work disability at baseline.

\section{Outcomes}

Information on sickness absence and permanent work disability was obtained from the Danish register on public transfer payments (DREAM) $(25,26)$, which includes weekly information on all public transfer payments to Danish citizens since 1996 (27). We focused on sickness absence $>2$ weeks because in the study period all employers could apply for a refund of sickness absence compensation after this length of time, which meant registration in DREAM. We defined permanent work disability as receiving flexible job subsidies or disability pension. Flexible job subsidies may be granted to persons whose work capacity is permanently reduced due to failing health. To be considered for disability pension, applicants must be permanently unable to work, even in a flexible job, for health reasons.

\section{Musculoskeletal pain}

We analyzed three mutually exclusive pain categories: no pain, limited pain [pain limited to the upper body (hands, wrists, forearms, elbows, shoulders, and/or neck) or lower body (feet, knees, hips, and/or lower back)], and combined pain (a combination of upper and lower body pain) (11). Pain was considered to be present if the participant answered at least "quite a lot" (response options: "not at all/very little/a little/somewhat/quite a lot/much/very much") to the question "How much have you been bothered by pain or discomfort in ... within the past 12 months?" or reported pain for $>30$ days within the past 12 months.

\section{Occupational mechanical exposures}

In order to assess occupational mechanical exposures to the upper and lower body, we used two job exposure matrices (JEM) $(28,29)$. The JEM were based on expert ratings of occupational mechanical exposures in the 2227 occupational titles, which are represented in the Danish version of the International Standard Classification of Occupations from 1988 (DISCO 88). We dichotomized upper-body exposures into a high and a low category. High upper-body exposures were assigned to participants with $\geq 1$ of the following exposures: a 
force-score $\geq 1$ indicating the mean force used at work on a scale from 0 (lowest) to 4 (highest) (30), upper-arm elevation $>90^{\circ}$ for $>45$ minutes/day, repetitive work with a frequency of $\geq 15$ movements of the upper arm per minute for $>30$ minutes/day, or repetitive work with a frequency of $\geq 4-15$ movements of the upper arm per minute for $>3$ hours/day (11). We dichotomized lower body exposures into a high and a low category using $\geq 1000$ kilograms lifted per day as a cut-off point (11). Participants were classified in the high category if either or both body regions were highly exposed.

We constructed a combined variable in order to examine the separate and combined influence of occupational mechanical exposures and musculoskeletal pain on the risk of the outcomes: (i) low mechanical exposures and no pain, (ii) low mechanical exposures and limited pain, (iii) low mechanical exposures and combined pain, (iv) high mechanical exposures and no pain, (v) high mechanical exposures and limited pain, and (vi) high mechanical exposures and combined pain.

\section{Occupational psychosocial exposures}

Occupational psychosocial exposures were assessed by questions concerning job demands, job control, and social support from leader and/or colleagues. Since the different studies used both single-item and multi-item scales for assessing psychosocial factors, we recoded the scales into a global scale ranging from 1 (best) to 6 (worst) (11). To avoid bias due to a tendency for those who experience pain to report more adverse exposures, we used a psychosocial JEM. The JEM was based on eight of the nine studies in the MRD as described elsewhere (11). In brief, we constructed a job group for each occupational title; if $<10$ participants held the same occupational title, they were included in the largest job group within the same or the numerically closest DISCO 88 code. We then calculated mean exposures for each job group. Job demands, job control, and social support were dichotomized based on the median; we kept the original dichotomization level in the present study. Job strain was based on the dichotomized variables for job demands and job control and categorized: no strain (low demands and high control, low demands and low control, or high demands and high control) and strain (high demands and low control).

We constructed a combined variable in order to examine the separate and combined influence of job strain and musculoskeletal pain on the risk of the outcomes: (i) no strain and no pain, (ii) no strain and limited pain, (iii) no strain and combined pain, (iv) strain and no pain, (v) strain and limited pain, and (vi) strain and combined pain.

\section{Covariates}

The following covariates were extracted from the MRD: age, sex, smoking, body mass index (BMI), and leisuretime physical activity (LTPA). One study (the project on cleaners and shop assistants) lacked information on smoking. In the remaining studies, smoking status was categorized: never smoker, ex-smoker, and current smoker. We categorized BMI as $<25, \geq 25-<30$, and $\geq 30 \mathrm{~kg} / \mathrm{m}^{2}$, and LTPA (participants were asked to mark the category that fitted best): low (moderate PA $<2$ hours/week), medium (moderate PA $\geq 2-\leq 4$ hours/week), and high (moderate PA $>4$ hours/week or strenuous $\mathrm{PA} \geq 2$ hours/week). We used information on unemployment insurance fund membership from DREAM to assess education level, which we categorized as higher- or medium-level, vocational, and low $(11,27)$. Information on insurance fund membership was missing for 539 persons, whose education level was assessed based on DISCO 88 codes.

\section{Statistical analysis}

Multivariable Cox regression analyses were performed. The results are reported as hazard ratios (HR) with 95\% confidence intervals (95\% CI). Follow-up started with the week following the completion of the baseline questionnaire and ended with the week of the respective outcome event or censoring at age 65, death, emigration, early voluntary retirement, or end of follow up after 52 weeks (sickness absence) or 104 weeks (permanent work disability), whichever came first. Combinations of (i) occupational mechanical exposures and pain and (ii) job strain and pain were analyzed in separate models with adjustment for job strain and occupational mechanical exposures, respectively. We a priori decided to include age (continuous), sex, BMI, LTPA, and social support in the multivariable analyses, while we decided only to include education level in supplementary analyses due to expected colinearity with the occupational exposures. We also subdivided the high category of occupational mechanical exposures into high upper- or lower-body exposures (ie, one but not both of high upper- and lower-body exposures, which almost exclusively meant upper-body exposures), or high upper- and lower-body exposures (11). Smoking was included in sensitivity analyses based on the studies, where this information was available. For all models, we ensured that the proportional hazards assumption was met by means of a global test, which also comprised a variable-by-variable test $(31,32)$. We used STATA 13 (StataCorp LP, College Station, TX, USA).

\section{Results}

Figure 1 shows the formation of the study cohort, which included 25292 persons: 14419 women and 10873 men. 


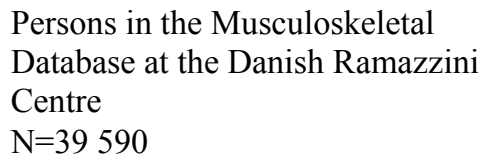

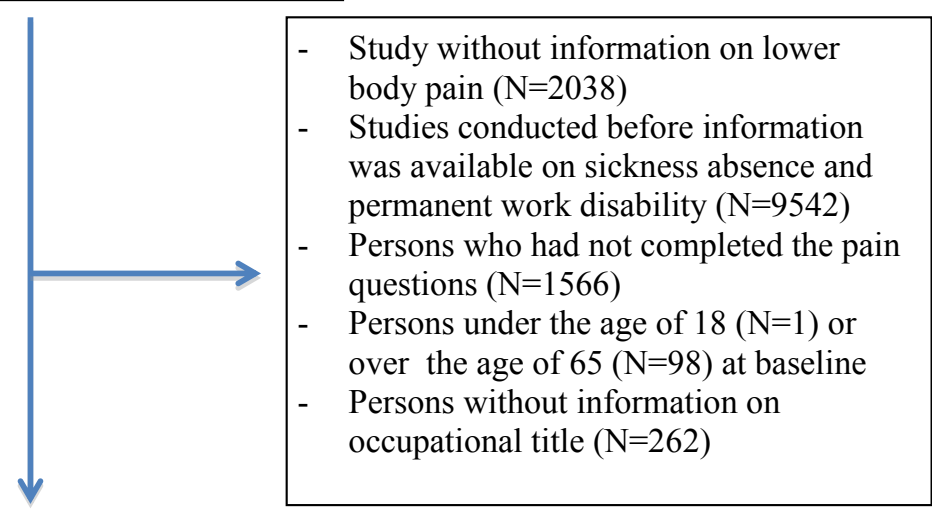
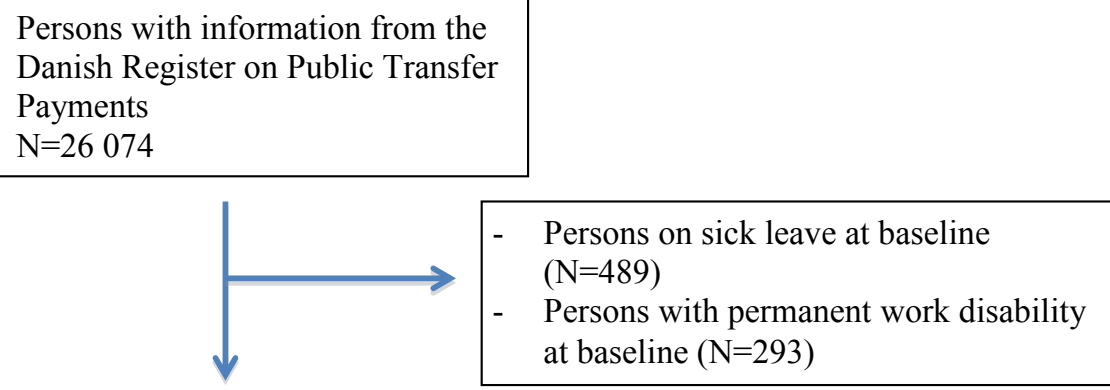

Persons eligible for follow-up

$\mathrm{N}=25292$

Table 1 displays characteristics of the study cohort according to combinations of occupational mechanical exposures and pain. Approximately $30 \%$ of the participants had high occupational mechanical exposures and approximately $10 \%$ were exposed to strain; within each stratum of occupational mechanical exposures, exposure to strain was evenly distributed across the three pain categories. The participants with high occupational mechanical exposures had the highest percentages with strain, low social support, and BMI in the two highest categories, and the lowest education level. The percentage of women increased with the extensiveness of pain both among those with low and those with high occupational mechanical exposures. For participants with low occupational mechanical exposures, the mean age was 42.7 years [standard deviation (SD) 10.1] for those with no pain, 42.9 (SD 9.8) years for those with limited pain, and 43.5 (SD 9.9) years for those with combined pain. For participants with high occupational mechanical exposures, the corresponding figures were 43.3 (SD 11.5), 44.4 (SD 11.1), and 46.5 (SD 10.6) years.

The participants were followed for 1231294 personweeks with respect to sickness absence and 2397615 person-weeks with respect to permanent work disability. During 52 weeks of follow-up, 2553 participants $(10 \%$ of the study cohort) were registered with a first-time sickness absence spell. During 104 weeks of follow-up, 119 participants $(0.5 \%)$ were registered with transition to permanent work disability.

Table 2 shows HR for sickness absence and permanent work disability in relation to combinations of occupational mechanical exposures and pain. For sickness absence, high mechanical exposures were associated with increased $\mathrm{HR}_{\mathrm{adj}}$ even in the group with no pain and the $\mathrm{HR}_{\text {adj }}$ increased with the extensiveness of pain both among those with low and among those with high exposures. The highest $\mathrm{HR}_{\text {adj }}$ were observed for the group with high exposures and combined pain. For permanent work disability, the pattern was similar. The $\mathrm{HR}_{\text {adj }}$ for (i) high mechanical exposures and limited pain and (ii) high mechanical exposures and combined pain were considerably lower than the crude HR, which was almost fully explained by adjustment for age (transfer payments due to permanent work disability are unlikely in young age, irrespective of exposures and pain), social support, and BMI. The $\mathrm{HR}_{\text {adj }}$ for strain was increased for 
sickness absence but not for permanent work disability. Low social support was associated with increased HR for both outcomes, in particular permanent work disability. BMI $\geq 30 \mathrm{~kg} / \mathrm{m}^{2}$ entailed increased HR, while high LTPA entailed reduced HR. For sickness absence, the $\mathrm{HR}_{\text {adj }}$ hardly changed when education level was included in the model (results not shown). For permanent work disability, it was impossible to include education level in the model because very few participants in the higher/ medium and vocational categories had high occupational mechanical exposures, while very few participants in the low category had low occupational mechanical exposures. When the high category of occupational mechanical exposures was subdivided into high upper- or lower-body exposures and high upper- and lower-body exposures, the $\mathrm{HR}_{\mathrm{adj}}$ for sickness absence in relation to combined pain were 3.15 (95\% CI 2.68-3.69) and 4.72 (95\% CI 3.98-5.60), respectively. The corresponding

Table 1. Characteristics of the study cohort according to combinations of occupational mechanical exposures and pain ( $\mathrm{N}=25$ 292). Numbers in cells are percentages. [BMI=body mass index; LTPA=leisure-time physical activity.]

\begin{tabular}{|c|c|c|c|c|c|c|}
\hline & \multicolumn{3}{|c|}{$\begin{array}{l}\text { Low occupational } \\
\text { mechanical exposures }\end{array}$} & \multicolumn{3}{|c|}{$\begin{array}{l}\text { High occupational } \\
\text { mechanical exposures }\end{array}$} \\
\hline & $\begin{array}{c}\text { No } \\
\text { pain } \\
(\mathrm{N}=8077)\end{array}$ & $\begin{array}{c}\text { Limited } \\
\text { pain a }^{a} \\
(\mathrm{~N}=5825)\end{array}$ & $\begin{array}{c}\text { Combined } \\
\text { pain }{ }^{b} \\
(\mathrm{~N}=4022)\end{array}$ & $\begin{array}{c}\text { No } \\
\text { pain } \\
(\mathrm{N}=2679)\end{array}$ & $\begin{array}{c}\text { Limited } \\
\text { pain }^{\mathrm{a}} \\
(\mathrm{N}=2305)\end{array}$ & $\begin{array}{c}\text { Combined } \\
\text { pain b } \\
(\mathrm{N}=2384)\end{array}$ \\
\hline \multicolumn{7}{|l|}{ Job strain } \\
\hline No strain & 92.0 & 92.6 & 91.5 & 81.0 & 77.9 & 78.9 \\
\hline Strain & 6.6 & 6.2 & 6.8 & 17.5 & 19.3 & 18.2 \\
\hline Missing & 1.4 & 1.3 & 1.6 & 1.6 & 2.8 & 2.9 \\
\hline \multicolumn{7}{|c|}{ Social support } \\
\hline High & 57.7 & 64.5 & 63.1 & 41.8 & 38.2 & 31.6 \\
\hline Low & 40.3 & 34.2 & 35.6 & 55.2 & 59.1 & 65.1 \\
\hline Missing & 2.0 & 1.4 & 1.3 & 3.0 & 2.7 & 3.4 \\
\hline \multicolumn{7}{|l|}{ Sex } \\
\hline Female & 52.8 & 61.6 & 70.8 & 39.9 & 50.9 & 61.6 \\
\hline \multicolumn{7}{|l|}{ BMI $\left(\mathrm{kg} / \mathrm{m}^{2}\right)$} \\
\hline$<25$ & 59.5 & 59.7 & 56.4 & 50.6 & 47.9 & 45.0 \\
\hline $25-<30$ & 31.1 & 29.9 & 30.6 & 36.0 & 35.2 & 37.6 \\
\hline$\geq 30$ & 7.3 & 8.5 & 10.5 & 10.6 & 13.5 & 14.3 \\
\hline Missing & 2.1 & 2.0 & 2.4 & 2.8 & 3.3 & 3.1 \\
\hline \multicolumn{7}{|l|}{ Smoking } \\
\hline Never & 50.0 & 46.2 & 41.5 & 39.7 & 32.9 & 25.4 \\
\hline Former & 25.0 & 27.2 & 28.4 & 22.5 & 21.0 & 20.3 \\
\hline Current & 24.5 & 26.2 & 29.5 & 33.0 & 32.2 & 32.6 \\
\hline Missing & 0.5 & 0.4 & 0.5 & 4.8 & 13.8 & 22.0 \\
\hline \multicolumn{7}{|l|}{ LTPA } \\
\hline Low & 14.1 & 16.0 & 18.1 & 15.4 & 16.6 & 16.7 \\
\hline Medium & 39.8 & 41.2 & 41.3 & 39.3 & 43.0 & 43.5 \\
\hline High & 45.3 & 42.2 & 39.8 & 42.3 & 37.5 & 34.8 \\
\hline Missing & 0.7 & 0.6 & 0.9 & 3.0 & 3.0 & 5.0 \\
\hline \multicolumn{7}{|l|}{$\begin{array}{l}\text { Education } \\
\text { level }\end{array}$} \\
\hline $\begin{array}{l}\text { Higher/ } \\
\text { medium }\end{array}$ & 35.9 & 28.6 & 24.8 & 11.3 & 8.8 & 7.2 \\
\hline Vocational & 58.0 & 65.0 & 67.2 & 39.0 & 34.1 & 27.5 \\
\hline Low & 6.2 & 6.4 & 8.0 & 49.7 & 57.1 & 65.4 \\
\hline
\end{tabular}

a Pain limited to the upper or lower body.

${ }^{\mathrm{b}} \mathrm{A}$ combination of upper and lower body pain.

$\mathrm{HR}_{\text {adj }}$ for permanent work disability were $3.64(95 \% \mathrm{CI}$ $1.74-7.62)$ and 6.96 (3.10-15.64). Sensitivity analyses, which included 22846 participants, showed $\mathrm{HR}_{\text {adj }}$ for current smoking of 1.30 (95\% CI $1.18-1.44)$ in relation to sickness absence and 1.23 (95\% CI $0.75-2.05)$ in relation to permanent work disability. Adjusting for smoking did not lead to any noticeable changes in the other results.

Table 3 displays HR of the outcomes in relation to combinations of job strain and pain. Strain was associated with sickness absence even in the group with no pain, but the association was weaker than for occupational mechanical exposures (cf. table 2). Again, the HR increased with the extensiveness of pain both among those with low and among those with high exposures, and the strongest associations were found for the group with strain and combined pain. Concerning permanent work disability, combined pain was the only significant

Table 2. Sickness absence and permanent work disability in relation to combinations of occupational mechanical exposures and pain. A total of 2553 participants received sickness absence benefits and 119 participants received flexible job subsidies or disability pension. [HR=hazard ratio; $95 \% \mathrm{Cl}=95 \%$ confidence interval; $\mathrm{BMI}=$ body mass index; LTPA=leisure-time physical activity.]

\begin{tabular}{|c|c|c|c|c|c|c|}
\hline & \multicolumn{3}{|c|}{ Sickness absence } & \multicolumn{3}{|c|}{ Permanent work disability } \\
\hline & $\mathrm{HR}_{\text {crude }}$ & $\mathrm{HR}_{\mathrm{adj}}{ }^{\mathrm{a}}$ & $95 \% \mathrm{Cl}$ & $\mathrm{HR}_{\text {crude }}$ & $H R_{a d j}{ }^{a}$ & $95 \% \mathrm{Cl}$ \\
\hline \multicolumn{7}{|c|}{$\begin{array}{l}\text { Combinations of } \\
\text { occupational mechanical } \\
\text { exposures and pain }\end{array}$} \\
\hline \multicolumn{7}{|l|}{$\begin{array}{l}\text { Low mechanical } \\
\text { exposures and }\end{array}$} \\
\hline No pain & 1.00 & 1.00 & .. & 1.00 & 1.00 & \\
\hline Limited pain ${ }^{b}$ & 1.43 & 1.38 & $1.21-1.58$ & 1.32 & 1.43 & $0.69-2.97$ \\
\hline Combined pain ${ }^{c}$ & 1.97 & 1.81 & $1.58-2.08$ & 3.65 & 3.35 & $1.74-6.45$ \\
\hline \multicolumn{7}{|c|}{$\begin{array}{l}\text { High mechanical } \\
\text { exposures and }\end{array}$} \\
\hline No pain & 2.22 & 2.16 & $1.86-2.51$ & 2.22 & 1.90 & $0.83-4.31$ \\
\hline Limited pain ${ }^{b}$ & 2.88 & 2.64 & $2.28-3.06$ & 2.22 & 1.15 & $0.44-3.03$ \\
\hline Combined pain ${ }^{c}$ & 4.15 & 3.71 & $3.24-4.25$ & 7.62 & 4.59 & $2.36-8.94$ \\
\hline \multicolumn{7}{|l|}{ Job strain } \\
\hline None & 1.00 & 1.00 & .. & 1.00 & 1.00 & \\
\hline Strain & 1.55 & 1.24 & $1.10-1.39$ & 1.17 & 1.00 & $0.60-2.01$ \\
\hline \multicolumn{7}{|l|}{ Social support } \\
\hline High & 1.00 & 1.00 &.. & 1.00 & 1.00 & \\
\hline Low & 1.50 & 1.30 & $1.19-1.41$ & 3.10 & 2.19 & $1.41-3.41$ \\
\hline Age & 1.00 & 0.99 & $0.99-1.00$ & 1.07 & 1.06 & $1.04-1.09$ \\
\hline \multicolumn{7}{|l|}{ Sex } \\
\hline Female & 1.00 & 1.00 & & 1.00 & 1.00 & \\
\hline Male & 0.80 & 0.75 & $0.69-0.82$ & 0.64 & 0.72 & $0.46-1.11$ \\
\hline \multicolumn{7}{|l|}{ BMI $\left(\mathrm{kg} / \mathrm{m}^{2}\right)$} \\
\hline$<25$ & 1.00 & 1.00 & & 1.00 & 1.00 & \\
\hline $25-<30$ & 1.11 & 1.08 & $0.99-1.19$ & 0.77 & 0.67 & $0.41-1.11$ \\
\hline$\geq 30$ & 1.64 & 1.44 & $1.27-1.62$ & 2.64 & 2.20 & $1.35-3.58$ \\
\hline \multicolumn{7}{|l|}{ LTPA } \\
\hline Low & 1.00 & 1.00 & & 1.00 & 1.00 & \\
\hline Medium & 0.86 & 0.90 & $0.80-1.01$ & 0.58 & 0.62 & $0.38-1.01$ \\
\hline High & 0.75 & 0.85 & $0.75-0.95$ & 0.46 & 0.49 & $0.29-0.83$ \\
\hline
\end{tabular}


Table 3. Sickness absence and permanent work disability in relation to combinations of job strain and pain. A total of 2553 participants received sickness absence benefits and 119 participants received flexible job subsidies or disability pension. [HR=hazard ratio; $95 \% \mathrm{Cl}=95 \%$ confidence interval]

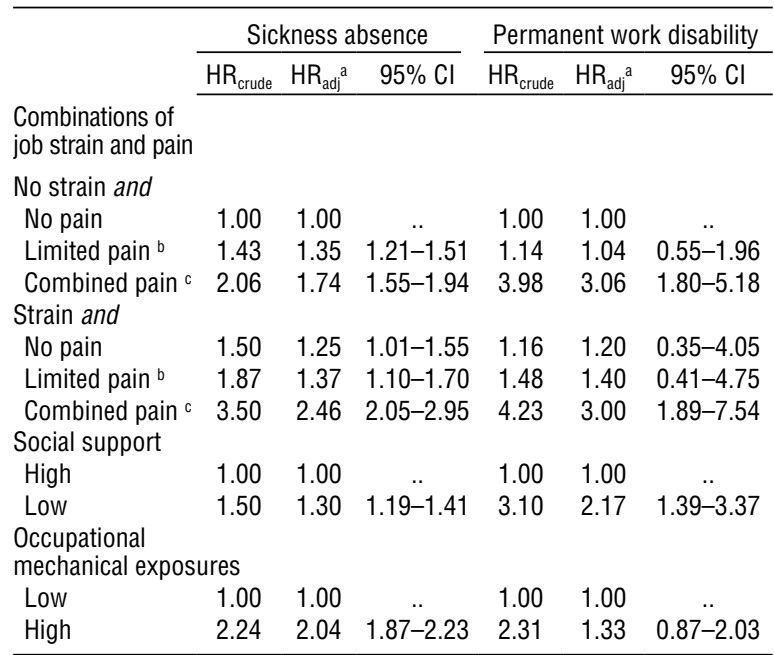

a Adjusted for all variables in the table and for age, sex, body mass index, and leisure-time physical activity.

${ }^{b}$ Pain limited to the upper or lower body.

${ }^{\mathrm{c}} \mathrm{A}$ combination of upper and lower body pain.

risk factor, and strain conveyed no additional risk. The $\mathrm{HR}_{\mathrm{adj}}$ for high mechanical exposures were considerably lower than the crude HR, which was almost fully explained by adjustment for age, social support, and BMI. Sensitivity analyses including smoking yielded results similar to those reported above.

\section{Discussion}

This study showed that combined pain per se was associated with a doubled risk of sickness absence and permanent work disability when compared to no pain. The $\mathrm{HR}_{\mathrm{adj}}$ increased with the extensiveness of pain both among those with low and those with high exposures. High occupational mechanical exposures per se were associated with a doubled risk of sickness absence and a similar, but insignificant increase in the risk of permanent work disability, while strain per se showed a modest association with sickness absence. All findings regarding combinations of exposures and pain were in accordance with a simple additive effect. Low social support at work was associated with an increased risk of sickness absence and especially permanent work disability.

This study benefited from a prospective design, complete follow up, and assessment of mechanical and psychosocial exposures by means of JEM, ie, independent from report of musculoskeletal pain. The use of
JEM averages out exposure contrasts to the extent that exposure variation within jobs is large as compared to the variation between jobs, but we did find contrasting exposures. It was a strength that both sickness absence and permanent work disability were studied so that the results could be compared, although it was a limitation that the number of transitions to permanent work disability was low. Another limitation was that the DREAM register does not contain information on medical reasons for sickness absence or permanent work disability. Participants may therefore have received transfer payments for reasons unrelated to musculoskeletal pain. To address this problem, we limited the follow-up period to 52 and 104 weeks for sickness absence and permanent work disability, respectively. To the extent that the transfer payments were unrelated to musculoskeletal pain, our effect estimates would be attenuated.

We defined sickness absence as $>2$ weeks based on the registration in DREAM in the study period. This definition has been used in previous studies of musculoskeletal pain in working populations $(16,17)$, but different definitions have been used in other studies, which we referred to in the introduction, ranging from $>3$ days (14), through $>6$ days (12), to $>8$ weeks $(13,15)$. Long-term sickness absence (often defined as $>10-14$ days) is generally believed to be more related to health problems than short-term sickness absence $(20,21)$. Instead of requiring longer periods of sickness absence, we included permanent work disability as a separate outcome.

Studies of widespread musculoskeletal pain often use the American College of Rheumatology's definition (33) or number of pain sites/multisite pain $(5,7,23,34)$. We were not able to adhere to these definitions, but participants with combined pain had similar characteristics to people with widespread or multisite pain with respect to somatization, illness worrying, and low Short Form 36 (SF-36) scores (11). We were unable to consider pain intensity, which has been related to sickness absence (16), but this may be of minor importance when the extensiveness of pain is taken into account (35).

Previous studies have found associations between number of pain sites and sickness absence $(5,9,20,23)$. This may reflect an effect of the number of pain sites per se, but additionally, the number of pain sites may represent an individual's propensity to be aware of and distressed by pain. Three of the just-mentioned studies addressed the influence of occupational exposures. The first study contrasted blue- and white-collar work and found that number of pain sites and blue-collar work were independently associated with subsequent sickness absence (20). High job strain was only associated with sickness absence in univariable analyses, and high social support was a preventive factor (20). The second study, which was cross-sectional, indirectly suggested 
that number of pain sites and physically hard work and/ or psychosocial exposures were independently related to sickness absence because the 3-fold increase in the prevalence of sickness absence, which was found for the group with multisite pain, increased to 3.6 after adjustment for these exposures, indicating negative confounding (5). The third study presented results for the extensiveness of pain stratified by duration of strenuous work, but did not provide estimates of the influence of duration of strenuous work per se; however, it could be seen that within each pain group, the mean number of sickness absence periods increased with the duration of strenuous work with up to a factor 1.6 when compared to the unexposed stratum (23). A similar pattern was not evident for job demands, job control, and social support. The association between the number of musculoskeletal pain sites and sickness absence was not modified by occupational exposures (23). Thus, our results were in agreement with the few previous studies on number of pain sites and sickness absence, except that previous studies have shown inconsistent results regarding social support.

We found that high occupational mechanical exposures per se were associated with a doubled risk of sickness absence and a similar but insignificantly increased risk of permanent work disability. This insignificant result probably reflects low statistical power; actually, the role of occupational mechanical exposures with respect to permanent work disability may be larger than the results suggest in so far as the causal pathway passes through musculoskeletal pain.

We think that our findings - together with the findings mentioned above $(5,20,23)$ - imply that primary and secondary preventive initiatives to minimize sickness absence should focus on reducing occupational mechanical exposures because this could reduce the necessity of sickness absence both directly and by lowering the occurrence of musculoskeletal pain. To the extent that occupational mechanical exposures lead to permanent work disability through musculoskeletal pain, the necessity of this outcome might be reduced as well. However, primary prevention may be a challenge for the workplaces because in many industries, success will require development of new task-specific innovations to reduce occupational mechanical exposures in addition to increased efforts to implement existing assistive devices. Our results point to persons, who develop musculoskeletal pain - in particular combined pain - while having a job with high mechanical exposures, as an obvious target group for secondary preventive efforts to reduce the exposures and thereby the necessity of sickness absence; this might also enable employees to keep working within the limitations of their impairments. Our finding that social support was particularly important with respect to permanent work disability suggests the same to the extent that social support implies workplace accom- modations. Regarding secondary prevention, general practitioners and other healthcare professionals should be aware that there is a need for efforts to reduce not only the number of musculoskeletal pain sites but also high occupational mechanical exposures. Thus, primary and secondary prevention efforts should be combined to reduce the necessity of sickness absence and permanent work disability.

Our results regarding sickness absence apply to periods lasting $>2$ weeks and may not be generalizable to short-term sickness absence. The baseline prevalence of occupational exposures and pain reflected the composition of the study cohort from the MRD, which cannot be considered representative of the Danish working population, and - like all studies of sickness absence and permanent work disability - our findings depended on national contexts with respect to access to transfer payments. Still, the results that point to factors which influence the necessity of these transfer payments should be generalizable, even across borders.

In conclusion, this study showed that combined pain in the upper and lower body was associated with sickness absence and permanent work disability and that high occupational mechanical exposures further increased the risk. Job strain showed a modest association with sickness absence, while low social support at work was associated with both outcomes, especially permanent work disability.

\section{Acknowledgements}

The Danish Working Environment Research Fund funded the establishment of the Danish Ramazzini Centre's MRD (Grant No. 31-2009-09).

\section{Conflicts of interest}

None declared.

\section{References}

1. Miranda H, Kaila-Kangas L, Heliövaara M, Leino-Arjas P, Haukka E, Liira J, et al. Musculoskeletal pain at multiple sites and its effects on work ability in a general working population. Occup Environ Med. 2010;67(7):449-55. http://dx.doi. org/10.1136/oem.2009.048249.

2. Parot-Schinkel E, Descatha A, Ha C, Petit A, Leclerc A, Roquelaure Y. Prevalence of multisite musculoskeletal symptoms: a French cross-sectional working populationbased study. BMC Musculoskelet Disord. 2012;13:122. http:// dx.doi.org/10.1186/1471-2474-13-122. 
3. Andersen LL, Mortensen OS, Hansen JV, Burr H. A prospective cohort study on severe pain as a risk factor for long-term sickness absence in blue- and white-collar workers. Occup Environ Med. 2011;68(8):590-2. http://dx.doi. org/10.1136/oem.2010.056259.

4. Coggon D, Ntani G, Palmer KT, Felli VE, Harari R, Barrero $\mathrm{LH}$, et al. Patterns of multisite pain and associations with risk factors. Pain. 2013;154(9):1769-77. http://dx.doi. org/10.1016/j.pain.2013.05.039.

5. de Fernandes RC, Burdorf A. Associations of multisite pain with healthcare utilization, sickness absence and restrictions at work. Int Arch Occup Environ Health. 2016 ;89(7):1047. http://dx.doi.org/10.1007/s00420-016-1141-7.

6. Gerdle B, Björk J, Cöster L, Henriksson K, Henriksson C, Bengtsson A. Prevalence of widespread pain and associations with work status: a population study. BMC Musculoskelet Disord. 2008;9:102. http://dx.doi.org/10.1186/1471-2474-9102.

7. Haukka E, Kaila-Kangas L, Ojajärvi A, Miranda H, Karppinen J, Viikari-Juntura E, et al. Pain in multiple sites and sickness absence trajectories: a prospective study among Finns. Pain. 2013;154(2):306-12. http://dx.doi.org/10.1016/j. pain.2012.11.003.

8. Kamaleri Y, Natvig B, Ihlebaek CM, Benth JS, Bruusgaard D. Change in the number of musculoskeletal pain sites: a 14-year prospective study. Pain. 2009;141(1-2):25-30. http://dx.doi. org/10.1016/j.pain.2008.09.013.

9. Nyman T, Grooten WJ, Wiktorin C, Liwing J, Norrman L. Sickness absence and concurrent low back and neck-shoulder pain: results from the MUSIC-Norrtälje study. Eur Spine J. 2007;16(5):631-8. http://dx.doi.org/10.1007/s00586-0060152-6.

10. Mortensen OS, Andersen JH, Ektor-Andersen J, Eriksen HR, Fallentin N, Frost P, et al. [White paper on sickness absence and return to work in people with musculoskeletal complaints. Causes and possible avenues for action.] Hvidbog om sygefravær og tilbgevenden til arbejde ved muskel- og skeletbesvær. Årsager og handlemuligheder. København: Det Nationale Forskningscenter for Arbejdsmiljø (NFA); 2008.

11. Sommer TG, Frost P, Svendsen SW. Combined musculoskeletal pain in the upper and lower body: associations with occupational mechanical and psychosocial exposures. Int Arch Occup Environ Health. 2015;88(8):99-110. http://dx.doi. org/10.1007/s00420-015-1036-z.

12. Labriola M, Lund T, Burr H. Prospective study of physical and psychosocial risk factors for sickness absence. Occup Med (Lond). 2006;56(7):469-74. http://dx.doi.org/10.1093/ occmed/kq1058.

13. Lund T, Labriola M, Christensen KB, Bültmann U, Villadsen E. Physical work environment risk factors for long term sickness absence: prospective findings among a cohort of 5357 employees in Denmark. BMJ. 2006;332(7539):449-52. http:// dx.doi.org/10.1136/bmj.38731.622975.3A.

14. Saastamoinen P, Laaksonen M, Lahelma E, Lallukka T, Pietiläinen O, Rahkonen O. Changes in working conditions and subsequent sickness absence. Scand J Work Environ Health. 2014;40(1):82-8. http://dx.doi.org/10.5271/ sjweh.3379.

15. Andersen LL, Clausen T, Persson R, Holtermann A. Doseresponse relation between perceived physical exertion during healthcare work and risk of long-term sickness absence. Scand J Work Environ Health. 2012;38(6):582-9. http://dx.doi. org/10.5271/sjweh.3310.

16. Holtermann A, Hansen JV, Burr H, Søgaard K. Prognostic factors for long-term sickness absence among employees with neck-shoulder and low-back pain. Scand J Work Environ Health. 2010;36(1):34-41. http://dx.doi.org/10.5271/ sjweh.2883.

17. Frost P, Haahr JP, Andersen JH. Impact of work, health and health beliefs on new episodes of pain-related and general absence-taking. Scand J Public Health. 2009;37(6):569-76. http://dx.doi.org/10.1177/1403494809341094.

18. Labriola M, Feveile H, Christensen KB, Strøyer J, Lund T. The impact of ergonomic work environment exposures on the risk of disability pension: prospective results from DWECS/ DREAM. Ergonomics. 2009;52(11):1419-22. http://dx.doi. org/10.1080/00140130903067771.

19. Lahelma E, Laaksonen M, Lallukka T, Martikainen P, Pietiläinen O, Saastamoinen P, et al. Working conditions as risk factors for disability retirement: a longitudinal register linkage study. BMC Public Health. 2012;12:309. http://dx.doi. org/10.1186/1471-2458-12-309.

20. Morken T, Riise T, Moen B, Hauge SH, Holien S, Langedrag $\mathrm{A}$, et al. Low back pain and widespread pain predict sickness absence among industrial workers. BMC Musculoskelet Disord. 2003;4:21. http://dx.doi.org/10.1186/1471-2474-421.

21. Nielsen ML, Rugulies R, Christensen KB, Smith-Hansen L, Kristensen TS. Psychosocial work environment predictors of short and long spells of registered sickness absence during a 2-year follow up. J Occup Environ Med. 2006;48(6):591-8. http://dx.doi.org/10.1097/01.jom.0000201567.70084.3a.

22. Mäntyniemi A, Oksanen T, Salo P, Virtanen M, Sjösten N, Pentti J, et al. Job strain and the risk of disability pension due to musculoskeletal disorders, depression or coronary heart disease: a prospective cohort study of 69,842 employees. Occup Environ Med. 2012;69(8):574-81. http://dx.doi. org/10.1136/oemed-2011-100411.

23. Neupane S, Pensola T, Haukka E, Ojajärvi A, eino-Arjas P. Does physical or psychosocial workload modify the effect of musculoskeletal pain on sickness absence? A prospective study among the Finnish population. Int Arch Occup Environ Health. 2016;89(5):719-28. http://dx.doi.org/10.1007/s00420-0151110-6.

24. Svendsen SW, Dalbøge A, Andersen JH, Thomsen JF, Frost P. Risk of surgery for subacromial impingement syndrome in relation to neck-shoulder complaints and occupational biomechanical exposures: a longitudinal study. Scand J Work Environ Health. 2013;39(6):568-77. http://dx.doi. org/10.5271/sjweh.3374. 
25. Hjøllund NH, Larsen FB, Andersen JH. Register-based followup of social benefits and other transfer payments: accuracy and degree of completeness in a Danish interdepartmental administrative database compared with a population-based survey. Scand J Public Health. 2007;35(5):497-502. http:// dx.doi.org/10.1080/14034940701271882.

26. Stapelfeldt CM, Jensen C, Andersen NT, Fleten N, Nielsen CV. Validation of sick leave measures: self-reported sick leave and sickness benefit data from a Danish national register compared to multiple workplace-registered sick leave spells in a Danish municipality. BMC Public Health. 2012;12:661. http://dx.doi. org/10.1186/1471-2458-12-661.

27. Svendsen SW, Frost P, Jensen LD. Time trends in surgery for non-traumatic shoulder disorders and postoperative risk of permanent work disability: a nationwide cohort study. Scand J Rheumatol. 2012;41(1):59-65. http://dx.doi.org/10.3109/0 3009742.2011.595375.

28. Dalbøge A, Hansson G-Å, Frost P, Andersen JH, HeilskovHansen T, Svendsen SW. Upper arm elevation and repetitive shoulder movements: a general population job exposure matrix based on expert ratings and technical measurements. Occup Environ Med. 2016;73(8):553-60. http://dx.doi.org/10.1136/ oemed-2015-103415.

29. Rubak TS, Svendsen SW, Andersen JH, Haahr JP, Kryger A, Jensen LD, et al. An expert-based job exposure matrix for large scale epidemiologic studies of primary hip and knee osteoarthritis: The Lower Body JEM. BMC Musculoskelet Disord. 2014;15:204. http://dx.doi.org/10.1186/1471-247415-204.
30. Moore JS, Garg A. The Strain Index: a proposed method to analyze jobs for risk of distal upper extremity disorders. Am Ind Hyg Assoc J. 1995;56(5):443-58. http://dx.doi. org/10.1080/15428119591016863.

31. Grambsch PM, Therneau TM. Proportional hazards tests and diagnostics based on weighted residuals. Biometrika. 1994;81(3):515-26. http://dx.doi.org/10.1093/ biomet/81.3.515.

32. Schoenfeld D. Partial residuals for the proportional hazards regression model. Biometrika. 1982;69(1):239-41. http:// dx.doi.org/10.1093/biomet/69.1.239.

33. Wolfe F, Smythe HA, Yunus MB, Bennett RM, Bombardier C, Goldenberg DL, et al. The American College of Rheumatology 1990 Criteria for the Classification of Fibromyalgia. Report of the Multicenter Criteria Committee. Arthritis Rheum. 1990;33(2):160-72. http://dx.doi.org/10.1002/ art. 1780330203 .

34. Haukka E, Kaila-Kangas L, Ojajärvi A, Saastamoinen P, Holtermann A, Jørgensen MB, et al. Multisite musculoskeletal pain predicts medically certified disability retirement among Finns. Eur J Pain. 2015;19(8):1119-28. http://dx.doi. org/10.1002/ejp.635.

35. Mose S, Christiansen DH, Jensen JC, Andersen JH. Widespread pain - do pain intensity and care-seeking influence sickness absence? - A population-based cohort study. BMC Musculoskelet Disord. 2016;17(1):197. http://dx.doi. org/10.1186/s12891-016-1056-1.

Received for publication: 2 August 2016 